\title{
Hagfish genome reveals parallel evolution of 7SL RNA-derived SINEs
}

Kenji K. Kojima

\begin{abstract}
Background: Short interspersed elements (SINEs) are ubiquitous components of eukaryotic genomes. SINEs are composite transposable elements that are mobilized by non-long terminal repeat (non-LTR) retrotransposons, also called long interspersed elements (LINES). The 3' part of SINEs usually originated from that of counterpart non-LTR retrotransposons. The $5^{\prime}$ part of SINEs mostly originated from small RNA genes. SINE1 is a group of SINEs whose $5^{\prime}$ part originated from 7SL RNA, and is represented by primate Alu and murine B1. Well-defined SINE1 has been found only from Euarchontoglires, a group of mammals, in contrast to the wide distribution of SINE2, which has a tRNA-derived sequence, from animals to plants to protists. Both Alu and B1 are mobilized by L1-type non-LTR retrotransposons, which are the only lineage of autonomous non-LTR retrotransposons active in these mammalian lineages.

Results: Here a new lineage of SINE1 is characterized from the seashore hagfish Eptatretus burgeri genome. This SINE1 family, designated SINE1-1_EBU, is young, and is transposed by RTE-type non-LTR retrotransposon, not L1-type. Comparison with other SINE families from hagfish indicated the birth of SINE1-1_EBU through chimera formation of a 7SL RNA-derived sequence and an older tRNA-derived SINE family. It reveals parallel evolution of SINE1 in two vertebrate lineages with different autonomous non-LTR retrotransposon partners. The comparison between two SINE1 lineages supports that the RNA secondary structure of the Alu domain of 7SL RNA is required for the efficient retrotransposition.

Conclusions: The hagfish SINE1 is the first evident SINE1 family found outside of Euarchontoglires. Independent evolution of SINE1 with similar RNA secondary structure originated in 7SL RNA indicates the functional importance of 7SL RNA-derived sequence in the proliferation of SINEs.
\end{abstract}

\section{Introduction}

Short interspersed elements (SINEs) are composite mobile elements that can mobilize dependent on the help of counterpart long interspersed elements (LINEs), also called non-long terminal repeat (non-LTR) retrotransposons $[1,2]$. SINEs are composed of several independently-originated regions, called head, body, and tail. These three regions are not always present in all SINEs, and sometimes, more than one independently derived sequences constitute one of these regions.

Correspondence: kojima@girinst.org

Genetic Information Research Institute, Cupertino, CA 95014, USA
The heads of SINEs typically originated from non-coding RNAs such as 7SL RNA, tRNA, 5S rRNA or small nuclear RNA (snRNA) [3, 4]. The heads serve primarily as internal promoters for the efficient transcription by RNA polymerase III [5]. SINEs with tRNA-originated heads, called SINE2, are the most common SINEs and widely distributed among eukaryotes [6]. SINEs with 5S rRNA-derived heads, called SINE3 are the second widely distributed and have been found from various vertebrates $[3,6]$. SINEs with 7SL RNA-derived heads are called SINE1. Well-defined examples of SINE1 are only found in one lineage of mammals, Euarchontoglires, which is composed of 5 orders: primates, flying lemurs, 
tree shrews, rodents, and lagomorphs [7]. Two of the best studied SINE families, Alu from humans and $B 1$ from mice, belong to SINE1. A putative SINE1 family was also found from marsupials [8], but this family, designated as P7SL_MD in Repbase, is composed of a fulllength 7SL RNA and a 3' poly A tail. It is structurally indistinguishable from retrocopies of 7SL RNAs. P7SL_ $M D$ was multiplicated into over 12,000 copies in the common ancestor of marsupials [9]. An expansion of putative retrocopies of 7SL RNA is also reported from the guinea pig genome [10].

$A l u$ is the abundant repetitive sequence accounting for $11 \%$ of the human genome [11]. A typical Alu sequence is a dimer composed by two 7SL RNA-derived monomer units connected by an A-rich linker [12]. Dimeric Alu elements are considered to have been originally generated through a fusion between free left Alu monomer (FLAM)-C and free right Alu monomer (FRAM) [13]. Two other types of Alu monomers, fossil Alu monomer $(F A M)$ and FLAM-A likely predated FLAM-C and FRAM $[14,15] . F L A M-A$ is nearly identical to the rodent SINE family $P B 1$ [16], and thus, FLAM-A/PB1 was likely born in the common ancestor of Euarchontoglires [7, 17]. Most rodents have families of monomeric 7SL RNA-derived SINEs called $B 1$ [18]. Tree shrews have Tu type SINEs, which show chimeric structures between 7SL RNA-derived SINEs and tRNA-derived SINEs [17]. SINE families originating by the fusion of tRNA-derived SINEs and 7SL RNA-derived SINEs were also found in the bushbaby Otolemur garnettii, designated as GarnAlu [19]. Active monomeric 7SL RNA-derived SINE families (Platy-1) were also found from the common marmoset Callithrix jacchus [20]. All SINE1 families found in Euarchontoglires are considered to be descendants of the common ancestor.

$A l u$ and $B 1$ are mobilized by the two proteins, L1ORF1p and L1ORF2p, encoded by $L 1[2,21] . L 1$ is distributed widely in eukaryotes [6]. Many mammals including humans and mice retain young $L 1$ lineages which are active or have been active recently. Unlike other non-LTR retrotransposons, mammalian L1 does not require the conserved RNA secondary structure in the 3' UTR for the recognition of the template RNA for reverse transcription [22]. Due to this relaxed recognition, $A l u$ and B1 RNAs as well as any polyadenylated mRNAs, can be mobilized by the $L 1$ machinery. Both $A l u$ and $B 1$ are composed solely by the sequences of 7SL RNA. The relaxed recognition by the $L 1$ machinery is also seen in plants, but it is considered that the machineries of non-mammalian $L 1$ as well as of other non-LTR retrotransposons recognize the RNA secondary structure besides the 3'-polyA tail $[1,23]$.

SINE1 families from Euarchontoglires share the sequence corresponding to the regions $1-63,76-83$, and
267-299 of the human 7SL RNA [7]. 7SL RNA-derived heads in SINE1 families from Euarchontoglires retain two functions. The internal promoter composed by two boxes (A box and B box), which is essential for the transcription of 7SL RNA, is located at 6-15 (A box) and at 76-86 (B box) in the human 7SL RNA [5]. 7SL RNA is a component of the signal recognition particle (SRP), which interacts with the ribosome. SINE1 families lack the central S domain of 7SL RNA, but retain the Alu domain composed by the $5^{\prime}$ and $3^{\prime}$ regions of 7SL RNA. The binding of Alu domain with SRP9/14 is required for the retrotransposition [24]. The binding of Alu RNA with SRP9/14 is proposed to be the mechanism of the efficient trans-mobilization by the $L 1$ machinery.

Here, a new SINE1 lineage is characterized from the seashore hagfish Eptatretus burgeri genome. This SINE1 family, designated SINE1-1_EBu, is young, and seems transposed by an RTE-type non-LTR retrotransposon family, not L1-type. It reveals parallel evolution of SINE1 in two vertebrate lineages with different autonomous non-LTR retrotransposon partners. The comparison between two SINE1 lineages indicates that the RNA secondary structure of the Alu domain is required for the efficient retrotransposition.

\section{Results \\ Identification of a novel SINE1 family from the seashore hagfish genome}

During the repeat analysis of the seashore hagfish genome, a repeat family was identified to show sequence similarity to the Alu families of SINEs. Refinement of repeat consensus sequence revealed that it is a SINE family whose $5^{\prime}$ region shows strong overlap to 7SL RNA genes (Fig. 1). It is designated SINE1-1_EBu as SINE1 refers a SINE family with 7SL RNA-derived head. The consensus sequence of SINE1-1_EBu is $282 \mathrm{bp}$ long. There are 2363 full-length insertions of SINE1-1_EBu in the hagfish genome, if excluding the $3^{\prime}$ microsatellites composed by AAC trinucleotides.

The 5' 96-bp sequence of SINE1-1_EBu shows strong sequence similarity to 7SL RNA genes in humans (Fig. $1 b)$. One copy of 7SL RNA gene was characterized from the hagfish genome (accession number FYBX02009602: 3123756-3,124,041), and it is more similar to SINE1-1 $E B u$ than to the human 7SL RNA gene. The predicted promoter box A in hagfish is 1 nucleotide different from that in the human genome (GGCGCAGTGG and GGCGCGGTGG; changes are in bold). The box B is different by 2 nucleotides between human and hagfish, AGTTCTGGGCT and AGCTCTGCGCT (changes are in bold), respectively.

The sequence 97-140 of SINE1-1_EBu is similar to the 3 ' terminus of 7SL RNA, while it shows less sequence similarity to the human 7SL RNA gene or SINE1 families 


\section{A}

1 ACCGGGCGTAGTGGCGTGCGCCTGTAATCCGGCTACTCGGAGGCTTGGGTTGGAGGATCGCTTGAGGACGGGAGCTCTGCGCTGGGCCGCGCTATGAGAG 100

101 GGTGTAGGGCAGCCCGGAATCATGGCGAAACCCAATCCTTATCCCTGTGTCGTAAGAGGCGACCAGGGTTGCGTCAGGAAGGGCATCCGGCGTAAAACCT 200

201 GTGCCAAATCAGTATGCGGATCATAACGGATGATCCGCTGTGGCGACCCCTAACGGGAGCAGCCGAAAGACGAACAACAACA 282

\section{B}

SINE1-1 EBU -ACCGGGCGTAGTGGCGTGCGCCTGTAAT-CCGGCTACTC-GGAGGCTTGGGTTGGAGGATCGCTTGAGGACGGGAGCTCTGCGCTGGGCCGCGCTATG----

7SLRNA_Hagfish GGCCGGGCGCAGTGGCGTGCGCCTGTAAT-CCGGCTACTC-GGAGGCTTGGGTTG-AGGATCGCTTGAGGACGGGAGCTCTGCGCTGTGCCGCGCTATGCC-7SLRNA Human GGCCGGGCGCGGTGGCGCGTGCCTGTAGTCCCAGCTACTCGGGAGGCT GAGGCTGGAGGATCGCTTGAGTCCAGGAGTTCTGGGCTGTAGTGCGCTATGCC-P7SL MD ----GGCATGGTGGCACATACCTGTAATCCCTGCTACTGGGGAGGCTGAGGCTGGTGGATCGCTTGAGTTCAGGAGTTCTGAGCTGCAGTAGGGCTAAAGAC P7.SL ${ }^{-} \mathrm{CpO}$-GCCGGGCGTGGTGGCGCGCGCCTGTAGTCCCAGCTACTCGGGAGGCTGAGGTGGGAGGATCGCTT GAGTCCAGGAGTTCTGGGCTGCAGTGCGCTATGCC-FRAM GGCCGGGCGCGGTGGCGCGCGCCTGTAGTCCCAGCTACTCGGGAGGCTGAGGCGGGAGGATCGCTTGAGCCCAGGAGTTCGAGGCTGCAGTGAGCTATGAT-$\begin{array}{lll}\text { FRAM } & \text { GGCCGGCGCGGTGGCGCGCGCCTGTAGTCCCAGCTACTCGGGAGGCTGAGGCGGGAGGATCGCTTGAGCCCAGGAGTTCGAGGCTGCAGTGAGCTATGAT-- } \\ \text { FAM } & \text { GGCGGGCGCGGTGGCGCGCGCCTGTAGTCCCAGTACTCGGGAGGCTGAGGCGGGAGGATGGCTTAGCCCAGGAGTTCGAGGCTGTAGTGCGTATGAT-- }\end{array}$

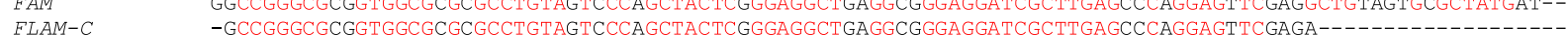

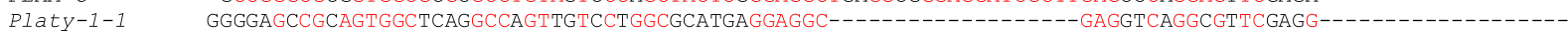

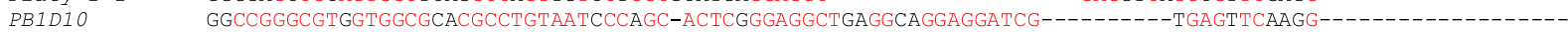

SINE1-1 EBU 7SLRNA_Hagfish -CGATCGGGCGTCCACGCCAAGTCCGGCATCGACATGGGGAGCCAGGGGGAGCCCTGCGCTCTCCAGGTCGCCTAAGGAGGGACGAAACGGCCCAGGCCGGCG 7SLRNA Human --GATCGGGTGTCCGCACTAAGTTCGGCATCAATATGGTGACCTCCCGGGAGCGGGGGAC-CACCAGGTTGCCTAAGGAGGGGTGAACCGGCCCAGGTCGGAA P7SL_MD C-AATTGGGTGTCTACACTAAGTCTGGCACCAATATGGTGAGCCCCTAGGAGTGGGGGGC-CACCAGGCTGCCTAAGGAGGGGTAAACTGGCCCAGGTCAGAA P7SL Cpo --GATCGGGTGTCCGCACTAAGTTCGGCATCAATATGGTGACCTCCCGGGAGCGGGGGAC-CACCAGGTTGCCTAAGGAGGGGTGAACCGGCCCAGGTCGGAA

FRAM

FAM $\quad$ FLM-C

Platy-1-1 --

PB1D10

SINE1-1 EBU

7SLRNA Hagfish ACGGAGCAGGTTAAAGTCCCCGTGCCGATCAGTGGTGGGATAGCGCCTGTGAATAGACGGTACAGA-GCAGCCCGGCCGATATGGCGGCACCCAACCCTT--

7SLRNA Human ACGGAGCAGGTCAAAACTCCCGTGCTGATCAGTAGTGGGATCGCGCCTGTGAATAGCCACTGCACT-CCAGCCTGGGCAACATAGCGAGACCCCGTCTCT--

P7SL_MD ATGGAGCAGGTCAAAGCTTCTGTGCT GATCAGTAGTGGGATCAGGCCCATGAGTGGCCACTGTACTTCCAGCCTGGGCAAGATAGGGAGACCCAGTCT (A) n

$\frac{\text { P7SL }}{-}$ Cpo ACGGAGCAGGTCAAAACTCCCGTGCTGATCAGTAGTGGGATCGCGCCTGTGAATAGCCACTGCACT-CCAGCCTGGGCAATATAGTGAGACCCTGTCTCT (A) ${ }_{n}$

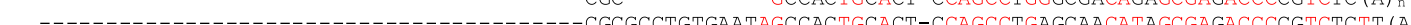

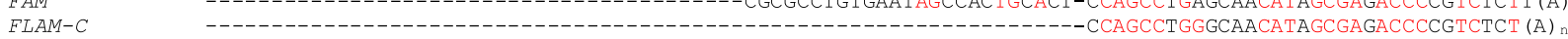

Platy-1-1 --

PB1D10 -

C

SINE1-1 EBU [137-277]

SINE2-1-EBu_[151-320]

SINE2-1B $E B$ S $_{-}[155-325$ ]

SINE2-9-EBu-[153-304]

SINE2-10_EBU_[149-342]

$R T E-4 E B \overline{B C}[1-125]$

RTE-2 $E B U-[1-137]$

RTE-2_EBU_[3085-3138]

-------CCGGTGTATGCCCCGGTTAGAATAGGTGTACCATATCCCT--GTGTCGTAAGAGGCGACCA----GGGTTG-CGTCAGGAA 作 -----CCGGTGTATGCCCCGGTTAGAATAGGTGTACCATATCCCTGTGTGTCGTAAGAGGTGACCA----GGGTTGGCGTCAGGAA ------CCGGTGTGTGCCCCGGTTAGAATAGGTACACCGTATCCCTGCGTGTCGTAAGAGGCGACTAAAAGGGTTGG-CGTCAGGAA AACCCAGCCACTGTGGTTGCACAAGTTAGTGCACTCTCAGCGCCGGTCC-CAAGCCCGGATAAATGGGGA----GGGTTG-CGTCAGGAA

SINE1-1_EBU_[137-277]

SINE2-1 EBu $[151-320]$

SINE2-1B EBu $[155-325]$

SINE2-9 EBR $[153-304]$

SINE2-10_EBu_[149-342]

$R T E-4=E B u_{-}[1-125]$

$R T E-2$ EBU $[1-137]$

$R T E-2$ EBU [ [3085-3138]

A

SINE1-1 EBu [137-279]

SINE2-1 EBu $[151-320]$

SINE2-1B B EBu $[155-325]$

$R T E-2$ EBu $[3 \overline{0} 85-3138]$

SINE2-9 EBU [153-304]

SINE2-10 EBU $[149-342]$

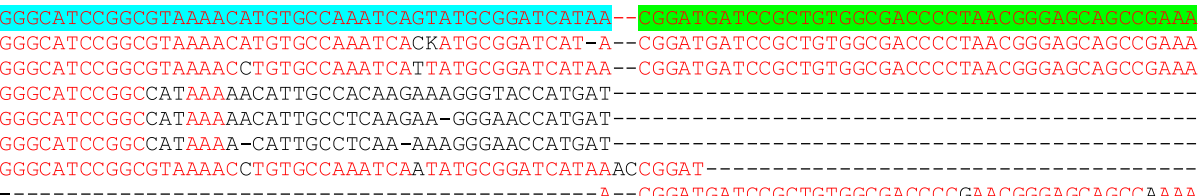

$R T E-4$ EBu $[$ [ $3 \overline{2} 83-3356$

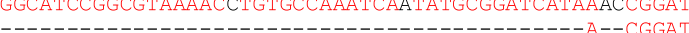

Fig. 1 Structure of SINE1-1_EBU. Segments showing similarity to different repeats are highlighted in different colors. Box A and Box B of RNA polymerase III promoter are in boldface. Nucleotides identical to SINE1-1_EBU consensus in alignments are colored in red. a Full-length sequence of SINE1-1_EBU. b Sequence alignment between SINE1-1_EBU, hagfish and human 7SL RNA genes and 7SL RNA-derived SINES from Euarchontoglires. One 7SL RNA gene from hagfish in the accession number FYBX02009602 is used for alignment. FRAM, FAM and FLAM-C are ancestral primate SINE1 families, and they are monomers. Platy-1-1 is a family from the common marmoset. PB1D10 is an ancestral rodent SINE family. c Sequence alignment between hagfish SINEs related to SINE1-1_EBU and their putative autonomous counterparts. Positions inside of entire consensus sequences are shown in parentheses 

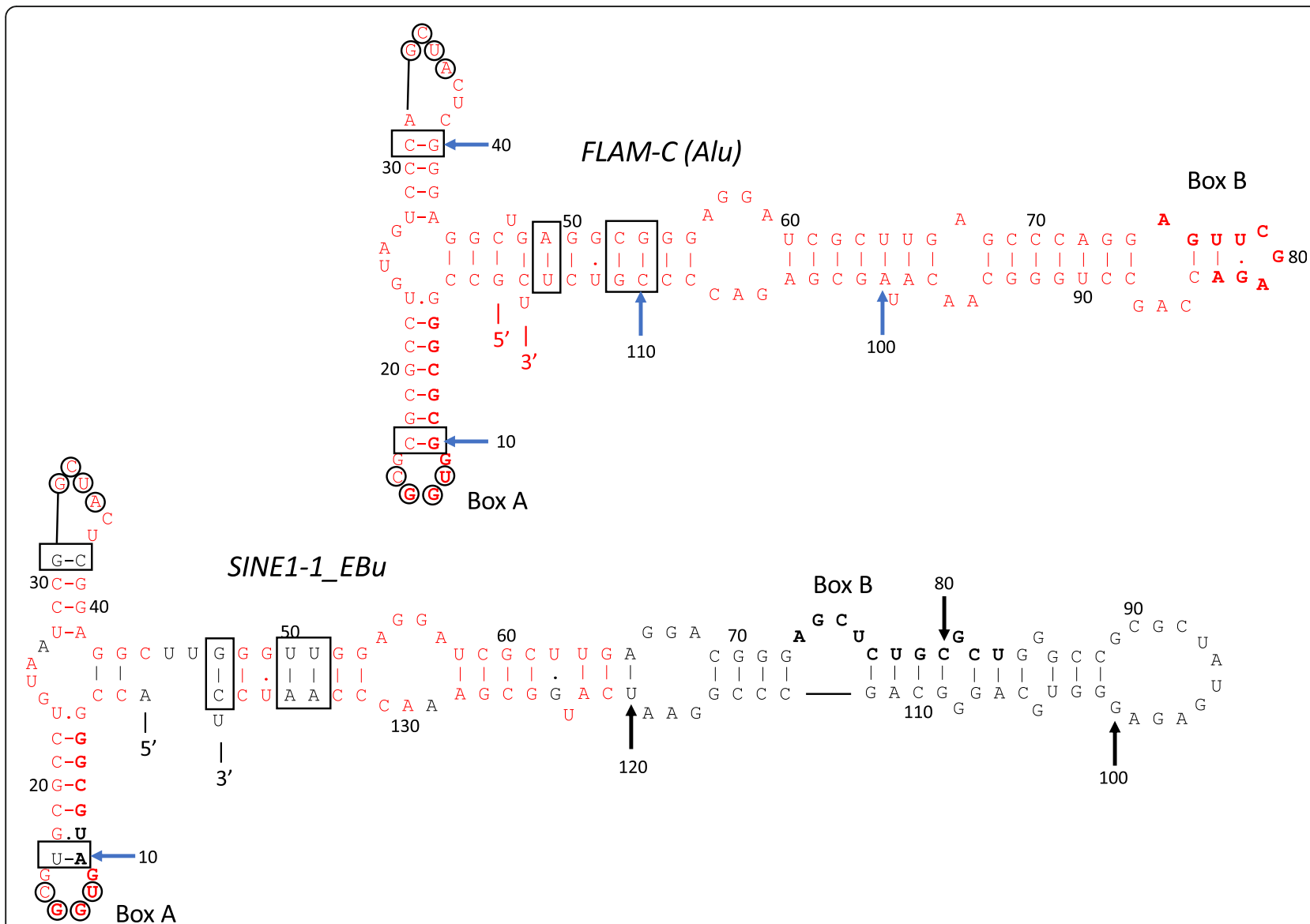

Fig. 2 Predicted secondary structures of 7SL RNA-derived regions of FLAM-C (the ancestor of left monomer of Alu) and SINE1-1_EBU. Nucleotides identical to the corresponding nucleotides in FLAM-C are colored in red in the structure of SINE1-1_EBU. Bases in the loops that form tertiary base pairs are circled. Compensatory substitutions are boxed. The nucleotides constituting the promoter box A and box B are in boldface

in Euarchontoglires (Fig. 1b). Despite their sequence differences, SINE1-1_EBu and FRAM show very similar lengths of deletions at the middle of 7SL RNA genes. The $5^{\prime}$ and the 3' regions of 7SL RNA constitute Alu domain. The predicted secondary structure of SINE1-1_EBu is consistent with the formation of Alu domain (Fig. 2). Compensatory substitutions of base-pairing nucleotides are seen and the overall structures are very similar between FLAM-C and SINE1-1_EBu. The ability to form $A l u$ domain is likely a sequence constraint for SINE1.

\section{SINE1-1_EBu appears mobilized by RTE-type non-LTR retrotransposons}

The 3' parts of SINE1-1_EBu shows some similarity to known RTE clade of non-LTR retrotransposons, such as RTE-9_LMi from the migratory locust Locusta migratoria and RTE-2 AFC from African cichlids (data not shown). Two families (RTE-2 EBu and RTE-4_EBu) of non-LTR retrotransposons which show $>90 \%$ sequence identity to the parts of SINE1-1_EBu, were reconstructed from $R T E$-related repeats in the RepeatModeler outputs
(Supplementary data S1). There are two and three fulllength copies of RTE-2_EBu and RTE-4_EBu in the hagfish genome, respectively, in addition to many fragmented copies. However, none of these copies encode intact proteins. Copies of RTE-2_EBu and RTE-4_EBu are $~ 93 \%$ identical to their respective consensus sequences.

SINE1-1_EBu was revealed to be a SINE having bipartite $R T E$-derived sequences (Fig. 1c). The sequence 165231 of SINE1-1_EBu is almost identical to a part of the 5' UTR of RTE-2_EBu, while the sequence 226-274 to the 3' end of RTE-2_EBu. Besides, the sequence 137-197 shows a high similarity to a part of the $5^{\prime}$ UTR of RTE4 $E B u$. These sequence similarities strongly support that SINE1-1_EBu is mobilized by $R T E-2 \_E B u$ or its closely related non-LTR retrotransposon family.

\section{Evolutionary relationships among SINEs and LINEs in the hagfish genome}

The average identity of the top 10 copies of SINE1-1 $E B u$ to the consensus is $\sim 99 \%$ and thus, SINE1-1_EBu is a young family. SINE1-1_EBu generates $\sim 17$-bp target 
site duplications (TSDs) upon integration, though the lengths of TSDs are not uniform (Supplementary Fig. S1A). Older copies also show similar lengths of TSDs if allowing a few nucleotide substitutions (data not shown). SINE1-1_EBu does not show strong target sequence preference. The full-length copies of RTE-2_EBu are flanked by 16 or 18-bp TSDs (Supplementary Fig. S1B), while the full-length copies of $R T E-4 \_E B u$ are by 6 or 19-bp TSDs (Supplementary Fig. S1C).

The sequence 137-274 of SINE1-1_EBu is similar to the sequence 198-337 of SINE2-1_EBu. SINE2-1_EBu has a longer sequence similar to $R T E-4 \_E B u$ than SINE1-1_EBu has (Fig. 1c). This similar sequence corresponds to the sequence 1-90 of RTE-4_EBu, while SINE1-1_EBu contains the sequence corresponding to the sequence 35-90 of RTE-4_EBu. The TSD length of SINE2-1_EBu is similar to those of SINE1-1_EBu, RTE2_EBu and RTE-4_EBu (Supplementary Fig. S1D).

SINE2-1_EBu has a tRNA-derived sequence at its 5' terminus (Fig. 3). The sequence following the tRNA-derived sequence shows similarity to UCON3. UCON3 was first found as an ultraconserved element shared among diverse vertebrate genomes [25]. Later, the similarity of UCON3 to a SINE family from chimaera, designated UCON3_CM, clarified that UCON3 is a part of SINE [26]. The position of sequence similar to UCON3 in SINE2-1_EBu is downstream of tRNA-derived sequence and upstream of $R T E$-derived sequence, indicating UCON3 is a type of conserved body, which is hereafter called UCON3 domain. Censor search against Repbase with UCON3 domain of SINE2-1_EBu as the query revealed SINE families in various animals, Chordata, Nematoda, Cnidaria, and Xenacoelomorpha, contain the conserved UCON3 domain (Supplementary Fig. S2).

\section{SINE families from hagfish show the SINE evolution through chimera formation}

Besides SINE2-1_EBu, 17 SINE families whose 5' termini show similarity to tRNAs were newly characterized (Fig. 3). Some families, such as SINE2-3_EBu, SINE23B_EBu and SINE2-3C_EBu, are closely related to each other with $>95 \%$ identity among their entire consensus sequences. It should be mentioned that they do not have parent-child relation or whole-part relation. They are independent SINE lineages, which shared their recent ancestor but transposed independently. The sequence alignment suggest that these tRNA-derived regions have several different origins (Supplementary Fig. S3). All SINE2 families found from the hagfish genome contain either of middle "body" regions: Deu, Meta, or UCON3 (Fig. 3 and Supplementary Figs. S2 and S4). EptSINE1 contains a tRNA-derived head and the middle Meta domain [27]. Comparison with newly characterized
EptSINE1B_EBu and EptSINE1C_EBu revealed that the original EptSINE1 sequence does not contain its 3' region. The 3' region of EptSINE1B_EBu and EptSINE1C_ $E B u$ shows sequence similarity to a newly characterized CR1-type non-LTR retrotransposon family, CR1-7_EBu (Supplementary Fig. S5). EbuSINE1 and EbuSINE2 contain a tRNA-derived head and a Deu domain at the middle, but their 3 ' regions show no sequence similarity to each other or to any non-LTR retrotransposons [28].

Some newly characterized hagfish SINE families show similarity to UCON3 (Supplementary Fig. S2). SINE families with UCON3 domain have distinct 3' tails. SINE2-1 $E B u$ and SINE2-1B_EBu contain RTE-type tail, almost identical to that of SINE1-1_EBu (Fig. 1c). SINE2-9_EBu and SINE2-10_EBu also contain RTE-type tails, but they contain the sequences similar to the $5^{\prime}$ UTR of RTE-4 $E B u$ and the 3' UTR of RTE-4_EBu (Fig. 1c). In other words, these two families of SINEs contain non-autonomous bipartite sequences derived from $R T E-4 \_E B u$.

SINE2-2_EBu, SINE2-2B_EBu and SINE2-2C_EBu have tails similar to that of EbuSINE2, despite the fact that EbuSINE2 contains a Deu domain upstream of the similar tail (Fig. 3 and Supplementary Figs. S4 and S5). SINE2-3_EBu and SINE2-3B_EBu have the tail similar to that of EbuSINE1, whereas EbuSINE1 contains a Deu domain (Fig. 3 and Supplementary Figs. S4 and S5). SINE2-4_EBu has a tail showing similarity to the 3' UTR of $C R 1-3 \_E B u$, and this tail shows similarity to the tails of SINE2-5_EBu and SINE2-5B_EBu, which have a Meta domain, and SINE2-7_EBu, which has a Deu domain (Fig. 3 and Supplementary Figs. S4 and S5). Compared with SINE families with either Meta domain or Deu domain, SINE families with UCON3 domain are younger. It is likely that the recombination between SINE families contributed to the birth of variation of SINE families with UCON3 domain.

\section{Age and evolution of hagfish SINE families}

Including SINE1-1_EBu, 21 SINE families were characterized from the inshore hagfish genome. The sequence identity of each copy to the consensus sequence is a measure of the age of family of transposable elements. The distributions of sequence identity to the consensus revealed that the youngest SINE family in the hagfish genome is SINE2-1_EBu, whose peak of identity distribution was between 97 and $96 \%$, followed by SINE1-1_EBu, SINE2-1B_EBu, and SINE2-3_EBu, all of whose peaks were between 96 and 95\% (Supplementary Fig. S6). It is also revealed that these 4 SINE families were concurrently active. The concurrent activities of SINE1-1_EBu, SINE2-1_EBu, and SINE2-1B_EBu are consistent with the fact that they appear transposed by the same autonomous non-LTR retrotransposon family, $R T E-2 \_E B u$. At the same time, the sequence similarity in 


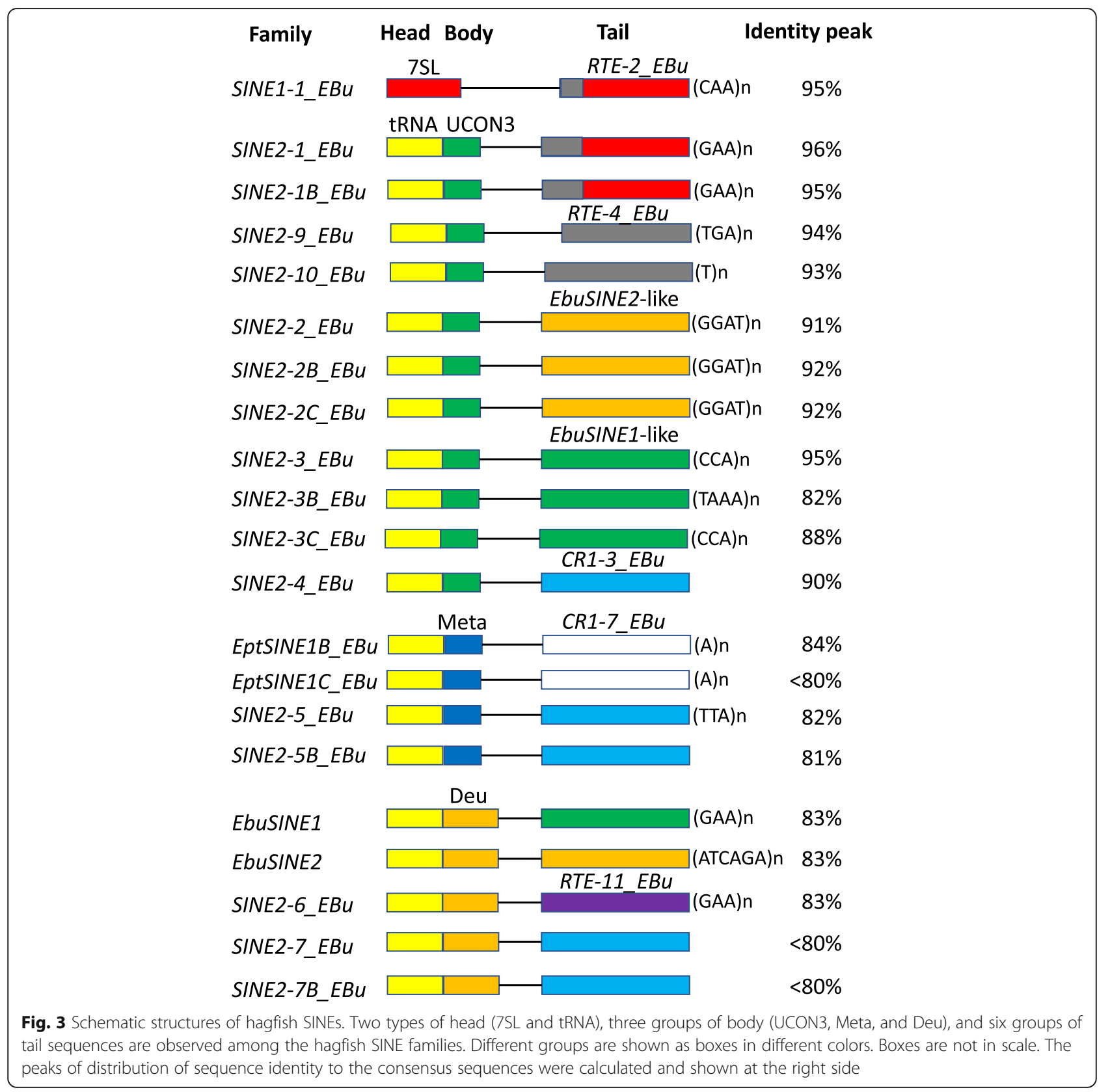

the 3 ' tail regions among several SINE families does not guarantee their concurrent activities. SINE2-3B_EBu, a relative of SINE2-3_EBu is very old and its peak was between 83 and $82 \%$. SINE2-3B_EBu and EbuSINE1 have similar 3 ' tails and were concurrently active. It indicates that autonomous non-LTR retrotransposon families were active for the long term with changing counterpart SINE families.

\section{Discussion}

SINE1-1_EBu is composed by 5 different parts (Fig. 1). The most $5^{\prime}$ region originated from the $5^{\prime}$ region of $7 \mathrm{SL}$ RNA, while the second $5^{\prime}$ region originated from the 3 ' region of 7SL RNA. These two parts are considered as the head of SINE1-1_EBu. The central part was derived from the 5' UTR of RTE-4_EBu. The two 3' regions originated from a non-autonomous RTE-2_EBu, corresponding to the $5^{\prime}$ and $3^{\prime}$ parts of $R T E-2 E B B u$. The latter three parts are considered as the tail of SINE1-1_ $E B u$, but the central part can also be considered as the body, considering its independent origin from the 3' two regions [29].

Based on the findings, it can be hypothesized how SINE1-1_EBu was born (Fig. 4). An autonomous nonLTR retrotransposon family related to RTE-4_EBu generated a non-autonomous derivative by the internal 


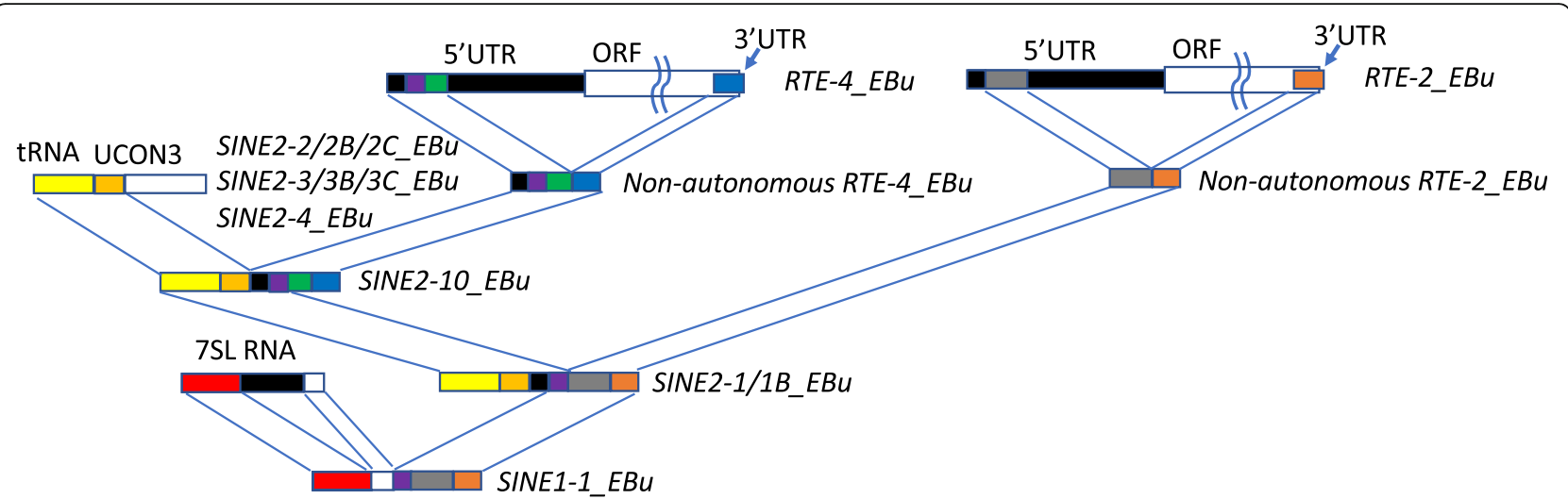

Fig. 4 A model of the birth of SINE1-1_EBu. Internal deletion of RTE-4_EBU and RTE-2_EBu generated non-autonomous bipartite retrotransposons. A chimeric SINE family, similar to SINE2-10_EBU, was generated from a non-autonomous RTE-4_EBu derivative and a SINE family having tRNA head and UCON3 domain. The 3' part of this SINE family was replaced by non-autonomous RTE-2_EBU derivative, which may be intact or a part of SINE, generating a SINE family similar to SINE2-1/1B_EBU. Finally, the chimera formation between a 7SL RNA and SINE2-1/1B_EBu-like SINE family generated SINE1-1_EBu. Internal deletion of 7SL RNA occurred either before or after the chimera formation. SINE1-1_EBu is composed by 5 parts: 5' region of 7SL RNA, 3' region of 7SL RNA, 5' region of RTE-4_EBU, 5' region of RTE-2_EBu, and 3' region of RTE-2_EBU

deletion. Similar event may have occurred with RTE-2 $E B u$. This type of bipartite non-autonomous retrotransposons are common for RTE-type non-LTR retrotransposons [29]. The chimera formation of nonautonomous, bipartite $R T E-4 \_E B u$ with a SINE2 family, possibly structurally related to $S I N E 2-2 / 2 B / 2 C \_E B u$, SINE2-3/3B/3C_EBu or SINE2-4_EBu, generated a SINE2 family which shows the same structure as SINE2$10 \_E B u$. Such chimera of two SINE families could be generated by DNA recombination. Switching template RNAs during retrotransposition is another possible mechanism of chimeric SINE formation [30]. This family would have been mobilized by RTE-4_EBu. The chimera formation between this SINE2-10_EBu-like SINE and a non-autonomous, bipartite $R T E-2 \_E B u$ generated a SINE family similar to SINE2-1/1B_EBu. The chimera formation between this SINE2-1/1B_EBu-like SINE and 7SL RNA generated a new SINE1 family. Internal deletion of 7SL RNA-derived region either followed or predated this event gave rise to SINE1-1_EBu. This scenario is simplified the most, and intermediate SINE or non-autonomous families, which have not yet characterized or have been lost completely from the genome, may have contributed to the birth of SINE1-1_EBu.

SINE1-1_EBu and SINE2-1/1B_EBu were concurrently active, which is expected since they appear mobilized by the same autonomous counterpart RTE-2_EBu. Slightly more recent activity of SINE2-1_EBu than SINE1-1_EBu does not contradict to the model, considering that relatively old copies of SINE2-1/1B_EBu could have contributed to the birth of SINE1-1_EBu.

RTE-type non-LTR retrotransposons often generate non-autonomous derivative families, which lack the internal portion of the autonomous counterpart. These bipartite non-autonomous RTE families sometimes generate chimeric retrotransposon families by acquiring $5^{\prime}$ head sequences originated from non-coding RNAs [29]. Most of such SINE families contain the sequence derived from tRNAs, and some contain the sequence from $5 S$ rRNAs. Two reported bird SINE families contain GC-rich heads of unknown origins upstream of bipartite RTE sequences [31]. One reported SINE family from budgerigar, called MeloSINE, has the $3^{\prime}$ end sequence of $28 \mathrm{~S}$ rRNA [31]. A SINE family called PlatSINE1 or snoRTE from platypus contains the sequence originated from snoRNAs at its $5^{\prime}$ end $[6,32]$. Their uniform chimeric structures among copies support their classification as SINE families. SINE1-1_EBu is the first reported SINE family containing a 7SL RNA-derived head and bipartite RTE sequences.

Theoretically, SINE1 can be born multiple times independently, as SINE2 and SINE3. Multiple independent events of birth of SINE2 are well supported by the different origins of tRNA-derived heads and the very wide distribution of SINE2 [6]. The alignment of head regions of hagfish SINE families also supports several independent origins for their heads (Supplementary Fig. S3). SINE2 families mobilized by various non-LTR retrotransposon families are reported [33]. SINE3 is less abundant. SINE3 was first found from zebrafish [3], and now it is known that SINE3 is present in various vertebrates and some insects (SINE3-1_TC from the red flour beetle Tribolium castaneum and HaSE3 from a moth Helicoverpa armigera, and their related SINE families) [6, 28, 34, 35]. Vertebrate SINE3 families are transposed by the CR1 clade of non-LTR retrotransposons, SINE3-1_TC seems mobilized by the $I$ clade of non-LTR retrotransposons, and HaSE3 seems 
mobilized by the RTE clade of non-LTR retrotransposons [29].

$A l u$ and $B 1$ are mobilized by $L 1$-type non-LTR retrotransposons [2, 21]. Regarding the nature of SINE mobilization by the transposition machinery of non-LTR retrotransposons, mammalian $L 1$ is an exception. Mammalian $L 1$ can mobilize any RNAs with poly A tail [2, 22], including cellular mRNA, RNA of endogenous retroviruses, or even RNA of RNA viruses [36-39]. The 3' ends of $A l u$ and $B 1$ are not similar to the $3^{\prime}$ ends of $L 1$ except poly A tails.

All SINEs with 7SL RNA-derived sequences found in euarchontoglires can be considered to be descendants of a single ancestral SINE1 family, born in the common ancestor of Euarchontoglires [7]. The ancestral SINE1 family could have resembled FLAM-A/PB1, which was an internally deleted derivative of 7SL RNA but had a poly A tail. The internal deletion as well as subsequent deletions/duplications distinguishes SINE families with 7SL RNA-derived sequences from retrocopies of 7SL RNA. P7SL_Cpo found from the guinea pig genome corresponds to the full-length 7SL RNA sequence followed by a polyA tail [10]. P7SL_Cpo should have originated independently from other SINE1 families, but the possibility that it corresponds to a set of retrocopies of 7SL RNA cannot be excluded. It is known that various types of small RNAs, such as small nuclear RNA (snRNA) or small nucleolar RNA (snoRNA) generate retrocopies which is composed of the full-length or partial RNA sequence and a 3'-poly A tail $[30,38,40]$. A proof that at least one copy of P7SL_Cpo is transposition-competent, is needed to establish the classification of P7SL_Cpo as a SINE1 family. P7SL_MD from marsupials is structurally almost identical to $P 7 S L_{-} C p o$ despite the sequence differences due to the divergence between these two groups of mammals $[8,9]$. There is not yet enough evidence for P7SL_Cpo and P7SL_MD to be recognized as SINE1 families.

It is obvious that SINE1-1_EBu was born independently from $A l u$ and B1. SINE1-1_EBu appears mobilized by $R T E$, based on the sequence similarity of the $3^{\prime}$ tail of SINE1-1_EBu with that of RTE-2_EBu. The internal deletion of 7SL RNA sequence in SINE1-1_EBu clearly excludes the possibility that it is a chimeric retrocopy of 7SL RNA and RTE-related non-LTR retrotransposons. Independent deletion events of the middle region of 7SL RNA, corresponding to the S domain, support no or little functional contribution of the $\mathrm{S}$ domain to the SINE proliferation. In contrast, the parallel conservations of base-pairing and the secondary structure of the Alu domain indicate the functional importance of the $A l u$ domain in the SINE proliferation. The conservation of Alu domain is indicated to be linked with the efficient inclusion of Alu RNA in the $L 1$ retrotransposition machinery [24]. Similar mechanism could have selected the conservation of Alu domain in the evolution of SINE1-1_ $E B u$.

\section{Conclusions}

The finding of SINE1-1_EBu, the first evident SINE1 family outside of Euarchontoglires, reveals the independent, parallel evolution of 7SL RNA-derived SINEs. The conservation of secondary structure of $A l u$ domain in independent SINE1 families indicates the functional importance of ternary structure of Alu domain bound to SRP9/14 in the proliferation of SINEs.

\section{Methods \\ Identification of SINE and LINE families from the seashore hagfish genome}

The genome sequence of inshore hagfish E. burgeri (Eburgeri_3.2) were downloaded from NCBI Assembly database (https://www.ncbi.nlm.nih.gov/assembly) on March 14, 2018. RepeatModeler (http://www.repeatmas ker.org/RepeatModeler/) and Repbase [6] were used for the initial screening of repetitive families with default parameters. Consensus sequences generated by RepeatModeler with the annotation for either SINE or LINE were chosen to reconstruct refined consensus sequences using the top 10 hits in the Censor search [41] with their 1000-bp flanking sequences at both sides. 7SL RNA gene sequences from the hagfish genome were found using the BLASTN searches using the 7SL RNA gene sequence from Ciona intestinalis (accession number: HG323729) as a query.

\section{Secondary structure prediction}

Secondary structure of $F L A M-C$ was predicted based on that of human 7SL RNA and AluY reported in [24]. Secondary structure of SINE1-1_EBu was predicted based on the sequence alignment and the secondary structure predicted at Web Servers for RNA Secondary Structure Prediction (https://rna.urmc.rochester.edu/RNAstructureWeb/).

\section{Supplementary information}

Supplementary information accompanies this paper at https://doi.org/10. 1186/s13100-020-00210-2.

Additional file 1: Figure S1. Target site duplications (TSDs). Figure S2. Sequence alignment of UCON3 domains of SINEs. Figure S3. Sequence alignment of tRNA-derived head regions of hagfish SINEs. Figure S4. Sequence alignments of body domains of hagfish SINEs. Figure S5. Sequence alignments of tails of hagfish SINEs. Figure S6. Age distribution of hagfish SINE families.

Additional file 2: Data S1. Consensus sequences of transposable elements characterized in this study.

\section{Abbreviations}

SINE: Short interspersed element; LTR: Long terminal repeat; snRNA: small nuclear RNA; SRP: Signal recognition particle; TSD: Target site duplication; RTE: Retrotransposon-like element 


\section{Authors' contributions}

KKK performed experiments and analysis, and wrote the manuscript. The author(s) read and approved the final manuscript.

\section{Funding}

None.

\section{Availability of data and materials}

All data generated or analyzed during this study are included in this published article and its supplementary information files. Consensus sequences of transposable elements are also available in Repbase (http:// www.girinst.org/repbase/).

\section{Ethics approval and consent to participate}

Not applicable.

\section{Consent for publication}

Not applicable.

\section{Competing interests}

The authors declare that they have no competing interests.

Received: 27 January 2020 Accepted: 27 March 2020

Published online: 22 May 2020

\section{References}

1. Kajikawa M, Okada N. LINEs mobilize SINEs in the eel through a shared 3' sequence. Cell. 2002;111(3):433-44.

2. Dewannieux M, Esnault C, Heidmann T. LINE-mediated retrotransposition of marked Alu sequences. Nat Genet. 2003;35(1):41-8.

3. Kapitonov W, Jurka J. A novel class of SINE elements derived from 5 S rRNA. Mol Biol Evol. 2003:20(5):694-702.

4. Kojima KK. A New Class of SINEs with snRNA Gene-Derived Heads. Genome Biol Evol. 2015;7(6):1702-12

5. Fuhrman SA, Deininger PL, LaPorte P, Friedmann T, Geiduschek EP. Analysis of transcription of the human Alu family ubiquitous repeating element by eukaryotic RNA polymerase III. Nucleic Acids Res. 1981;9(23):6439-56.

6. Bao W, Kojima KK, Kohany O. Repbase Update, a database of repetitive elements in eukaryotic genomes. Mob DNA. 2015;6:11.

7. Kriegs JO, Churakov G, Jurka J, Brosius J, Schmitz J. Evolutionary history of 7SL RNA-derived SINEs in Supraprimates. Trends Genet. 2007;23(4):158-61.

8. Gentles AJ, Wakefield MJ, Kohany O, Gu W, Batzer MA, Pollock DD, et al. Evolutionary dynamics of transposable elements in the short-tailed opossum Monodelphis domestica. Genome Res. 2007;17(7):992-1004.

9. Nilsson MA, Janke A, Murchison EP, Ning Z, Hallstrom BM. Expansion of CORE-SINEs in the genome of the Tasmanian devil. BMC Genomics. 2012;13: 172

10. Bao W, Jurka J. P7SL_Cpo, a 7SL pseudogene from the guinea pig. Repbase Reports. 2011;11(5):1736.

11. Lander ES, Linton LM, Birren B, Nusbaum C, Zody MC, Baldwin J, et al. Initial sequencing and analysis of the human genome. Nature. 2001;409(6822): 860-921.

12. Ullu E, Tschudi C. Alu sequences are processed 7SL RNA genes. Nature. 1984:312(5990):171-2.

13. Quentin Y. Fusion of a free left Alu monomer and a free right Alu monome at the origin of the Alu family in the primate genomes. Nucleic Acids Res. 1992;20(3):487-93.

14. Quentin Y. Origin of the Alu family: a family of Alu-like monomers gave birth to the left and the right arms of the Alu elements. Nucleic Acids Res. 1992;20(13):3397-401.

15. Jurka J, Zuckerkandl E. Free left arms as precursor molecules in the evolution of Alu sequences. J Mol Evol. 1991;33(1):49-56.

16. Quentin Y. A master sequence related to a free left Alu monomer (FLAM) at the origin of the B1 family in rodent genomes. Nucleic Acids Res. 1994; 22(12):2222-7.

17. Nishihara H, Terai Y, Okada N. Characterization of novel Alu- and tRNArelated SINEs from the tree shrew and evolutionary implications of their origins. Mol Biol Evol. 2002;19(11):1964-72.

18. Veniaminova NA, Vassetzky NS, Kramerov DA. B1 SINEs in different rodent families. Genomics. 2007;89(6):678-86.
19. Jurka J. SINE elements from the bushbaby genome. Repbase Reports. 2010; 10(5):778-80.

20. Konkel MK, Ullmer B, Arceneaux EL, Sanampudi S, Brantley SA, Hubley R, et al. Discovery of a new repeat family in the Callithrix jacchus genome. Genome Res. 2016;26(5):649-59.

21. Dewannieux M, Heidmann T. L1-mediated retrotransposition of murine B1 and B2 SINEs recapitulated in cultured cells. J Mol Biol. 2005;349(2):241-7.

22. Okada N, Hamada M, Ogiwara I, Ohshima K. SINEs and LINEs share common 3' sequences: a review. Gene. 1997:205(1-2):229-43.

23. Ohshima K. Parallel relaxation of stringent RNA recognition in plant and mammalian L1 retrotransposons. Mol Biol Evol. 2012;29(11):3255-9.

24. Ahl V, Keller H, Schmidt S, Weichenrieder O. Retrotransposition and Crystal Structure of an Alu RNP in the Ribosome-Stalling Conformation. Mol Cell. 2015;60(5):715-27

25. Jurka J, Kohany O. UCON3: Conserved interspersed repeat from mammals and birds. Repbase Reports. 2006;6(10):534.

26. Jurka J. A conserved SINE element from the elephant shark: consensus. Repbase Reports. 2016;16(12):2222.

27. Nishihara H, Plazzi F, Passamonti M, Okada N. MetaSINEs: Broad Distribution of a Novel SINE Superfamily in Animals. Genome Biol Evol. 2016;8(3):528-39.

28. Nishihara H, Smit AF, Okada N. Functional noncoding sequences derived from SINEs in the mammalian genome. Genome Res. 2006;16(7):864-74.

29. Kojima KK. LINEs Contribute to the Origins of Middle Bodies of SINEs besides 3' Tails. Genome Biol Evol. 2018;10(1):370-9.

30. Buzdin A. The human genome contains many types of chimeric retrogenes generated through in vivo RNA recombination. Nucleic Acids Res. 2003; 31(15):4385-90.

31. Suh A, Witt CC, Menger J, Sadanandan KR, Podsiadlowski L, Gerth M, et al. Ancient horizontal transfers of retrotransposons between birds and ancestors of human pathogenic nematodes. Nat Commun. 2016;7:11396.

32. Schmitz J, Zemann A, Churakov G, Kuhl H, Grutzner F, Reinhardt R, et al. Retroposed SNOfall--a mammalian-wide comparison of platypus snoRNAs. Genome Res. 2008:18(6):1005-10.

33. Ohshima K. RNA-Mediated Gene Duplication and Retroposons: Retrogenes, LINEs, SINEs, and Sequence Specificity. Int J Evol Biol. 2013;2013:424726.

34. Kapitonov W, Jurka J. SINE3-1_TC, a family of SINE3 retrotransposons from the red flour beetle genome. Repbase Reports. 2007:7(11):1180.

35. Wang J, Wang A, Han Z, Zhang Z, Li F, Li X. Characterization of three novel SINE families with unusual features in Helicoverpa armigera. PLoS One. 2012;7(2):e31355.

36. Horie M, Honda T, Suzuki Y, Kobayashi Y, Daito T, Oshida T, et al. Endogenous non-retroviral RNA virus elements in mammalian genomes. Nature. 2010;463(7277):84-7.

37. Esnault C, Maestre J, Heidmann T. Human LINE retrotransposons generate processed pseudogenes. Nat Genet. 2000;24(4):363-7.

38. Kojima KK. Different integration site structures between L1 proteinmediated retrotransposition in cis and retrotransposition in trans. Mob DNA. 2010;1(1):17.

39. Schmitz J, Churakov G, Zischler H, Brosius J. A novel class of mammalianspecific tailless retropseudogenes. Genome Res. 2004;14(10A):1911-5.

40. Doucet AJ, Droc G, Siol O, Audoux J, Gilbert N. U6 snRNA Pseudogenes: Markers of Retrotransposition Dynamics in Mammals. Mol Biol Evol. 2015; 32(7):1815-32

41. Kohany O, Gentles AJ, Hankus L, Jurka J. Annotation, submission and screening of repetitive elements in Repbase: RepbaseSubmitter and Censor. BMC Bioinformatics. 2006;7:474

\section{Publisher's Note}

Springer Nature remains neutral with regard to jurisdictional claims in published maps and institutional affiliations. 